\title{
A New Adaptive System for Suppression of Transient Wind Disturbance in Large Antennas
}

\author{
Wei Liang $\left(\mathbb{D}\right.$, Jin Huang $\mathbb{D}^{D}$, and Jie Zhang $(\mathbb{D}$ \\ Key Laboratory of Electronic Equipment Structure Design, Ministry of Education, Xidian University, Xi'an 710071, China \\ Correspondence should be addressed to Jin Huang; jhuang@mail.xidian.edu.cn and Jie Zhang; jiezhang1987@xidian.edu.cn
}

Received 15 January 2019; Accepted 29 July 2019; Published 4 September 2019

Academic Editor: Hervé Aubert

Copyright (c) 2019 Wei Liang et al. This is an open access article distributed under the Creative Commons Attribution License, which permits unrestricted use, distribution, and reproduction in any medium, provided the original work is properly cited.

\begin{abstract}
Under transient wind disturbance, vibration deformation of the large antenna surface profile causes deterioration of pointing performance. This paper presents a new adaptive system to suppress unknown transient wind disturbance. First, to monitor the vibration, based on the acceleration measurement and a low-order flexible model considering equivalent identification of forces, the real-time estimation of the vibration state is obtained in an unbiased minimum-variance way. Next, a novel fourcable-actuator mechanism with a circular slide track is proposed for suppressing the vibration, in which the locations of the cable drivers on the slide track are determined according to the attitude of the antenna, and the expected tension distribution of the cables is found by the vibration state and the optimal gain of the linear quadratic regulator (LQR). In the end, the simulation implementation of a $7.3 \mathrm{~m}$ antenna under the transient wind disturbance is used to verify the effectiveness of the proposed method.
\end{abstract}

\section{Introduction}

Large antennas are widely used in deep space exploration, satellite communications, radio astronomy, and other fields. With the development trend of the large-aperture and high-frequency bands for reflector antennas, a higher pointing accuracy is required under such conditions. For the European Space Agency designed $35 \mathrm{~m}$ deep space antenna, a pointing accuracy of $0.006^{\circ}$ is required in order to operate it at $35 \mathrm{GHz}$ [1]. As large reflector antennas have a low natural frequency and primarily operate in open air without radome, stochastic wind disturbance obviously increases structural vibration deformation (SVD) which results in pointing performance degradation [2]. To reduce the pointing error (PE), the first step should be to measure the SVD and then to control and compensate for it.

There are many measurement methods to get the SVD. But it is very difficult for a large antenna because conventional contact-type transducers require fixed reference points which are rarely found in real situations [3]. Furthermore, the attitude of a large antenna is different during the working process. Although noncontact photogrammetry and laser-based transducers can sense relative displacement [4], these instruments are also incapable of the whole working condition measurement because of light obstruction. On the contrary, accelerometers can satisfy the requirements of the measurements of multiple points without fixed reference points and light obstruction. For reconstructing displacement from acceleration, the frequency-domain integration approach (FDIA) and two integrators with a high-pass digital filter are most commonly used [5]. In references [6, 7], some accelerometers were placed on the antenna reflector to study the dynamic behaviour of the antenna based on the FDIA under the transient wind disturbance. However, the conventional integration approaches need to remove the pseudostatic and low-frequency signals to overcome the integral drift. Moreover, if the FDIA driven by FFT and IFFT is executed in a relatively short time window, the method causes 
serious discretization errors [5]. Therefore, they are the major drawbacks for complete displacement reconstruction in real time.

And to control and compensate for the PE caused by wind disturbance, several control schemes have been developed in large antennas. These methodologies can be roughly divided into two categories. The first category involves using the fixed compensation with the lookup table. For example, Ukita proposed an auxiliary pointing correction system using a lookup table, in which data are compiled from all sky-pointing measurements under different wind conditions. This method was able to reduce the RMS PE from 2.4 to 1.2 arcsec at a static wind speed of $9.3 \mathrm{~m} / \mathrm{s}$ [6]. However, the method is inconvenient for application because a large amount of data need to be collected, and it only focuses on static wind. The second category is to consider the wind spectrum characteristics in the controller design of the hinge servo system. Using three wind models, Gawronski applied various control theories such as PID, LQG, and $H_{\infty}$ and discussed their basic properties, tracking precision, and limitations [8]. Qiu proposed an extended state observer using the Davenport wind spectrum [9]. These methods have a certain amelioration in suppressing transient wind disturbance. However, the category only adjusts the PE obtained by the angle encoders, the serious SVD-induced PE, that is, the dominant source of the total PE in large antennas is ignored [10]. So a pointing error analytical model was proposed by Zhang to estimate the pointing error caused by the antenna structure deformation [11]. Based on the model, Zhang used different controller design methods such as the model predictive controller [12], the linear-quadratic-Gaussian controller, and the sliding mode controller [13] to compensate for the SVDinduced PE via a rotating shaft servo system. However, the SVD of the antenna is not suppressed. As a result, there is no effective control method to reduce SVD of a large antenna under transient wind disturbance.

In response to the above problems, a new adaptive system is proposed to suppress transient wind disturbance in large antennas. This paper is organized as follows: In Section 2, the adaptive system is outlined. Next, in Sections 3, a low-order flexible antenna model under transient wind forces and cable tensions in a three-dimensional space is introduced. Section 4 presents a real-time estimator of the vibration state based on acceleration measurements and the low-order flexible model considering equivalent identification of force. The cable tension controller to suppress the vibration based on the LQR is proposed in Section 5. In Section 6, the simulation example is undertaken. Finally, in Section 7, the work is summarized.

\section{Synthetic Design Scheme with Integration of Mechanical, Sensing, and Control Technologies}

The adaptive system is composed of a vibration monitoring and control system, as shown in Figure 1. The accelerometers placed on the reflector are used to obtain the vibration state of SVD caused by wind forces and tensions of the cables. And for the convenience of analysis and implementation, the four-cable-actuator mechanism with a circular slide track is designed to suppress unknown transient wind forces; the actual tension is measured by the tension sensor. The state estimator of vibration and tension controller of cables are discussed in Sections 4 and 5, respectively.

As shown in Figures 1 and 2, the center of the slide track with radius $R_{2}$ is located at the center of the antenna base. The drivers can move on the slide track with the change of the azimuth and elevation angles of the antenna. When the azimuth angle is fixed, the " 1 " and " 3 " drivers are located at the intersection of the $X$-axis and the slide track, and the distance between the " 2 " or " 4 " driver and the $Y$-axis is $L$. Then, the coordinate of the drivers is found, as shown in Figure 2:

$$
L=d \cos E_{m}+l,
$$

where $E_{m}$ is the elevation angle, $d$ is the distance between the elevation axis and the antenna aperture surface, and $l$ is the distance between the azimuth axis and the elevation axis.

The four tensions act on the edge of the antenna aperture surface with radius $R_{1}$. The connection line between action points of $T_{1}$ and $T_{3}$ is perpendicular to the elevation axis, and the vectors of $T_{1}$ and $T_{3}$ are located in the $X Z$ plane; the connection line between action points of $T_{2}$ and $T_{4}$ is parallel to the elevation axis, and the vectors of $T_{2}$ and $T_{4}$ are located in a plane perpendicular to the $X Y$ plane.

The heights of action points of $T_{i}(i=\{1,2,3,4\})$ are

$$
\begin{aligned}
& H_{1}=H_{2}-R_{1} \cos E_{m}, \\
& H_{2}=H_{4}=d \sin E_{m}+h, \\
& H_{3}=H_{2}+R_{1} \cos E_{m},
\end{aligned}
$$

where $h$ is the height of the elevation axis.

The angles between $T_{i}$ and negative $Z$-axis are

$$
\begin{aligned}
& \alpha_{1}=\arctan \left(\frac{R_{2}-\left(R_{1} \sin E_{m}+L\right)}{H_{1}}\right), \\
& \alpha_{2}=\alpha_{4}=\arctan \left(\frac{\sqrt{R_{2}^{2}-L^{2}}-R_{1}}{H_{2}}\right), \\
& \alpha_{3}=\arctan \left(\frac{R_{2}+L-R_{1} \sin E_{m}}{H_{3}}\right) .
\end{aligned}
$$

Components of $T_{i}$ along $X, Y$, and $Z$ directions are

$$
\begin{aligned}
& T_{1 x}=T_{1} \sin \alpha_{1} ; T_{1 y}=0 ; T_{1 z}=-T_{1} \cos \alpha_{1}, \\
& T_{2 x}=0 ; T_{2 y}=T_{2} \sin \alpha_{2} ; T_{2 z}=-T_{2} \cos \alpha_{2}, \\
& T_{3 x}=-T_{3} \sin \alpha_{3} ; T_{3 y}=0 ; T_{3 z}=-T_{3} \cos \alpha_{3}, \\
& T_{4 x}=0 ; T_{4 y}=-T_{4} \sin \alpha_{4} ; T_{4 z}=-T_{4} \cos \alpha_{4} .
\end{aligned}
$$




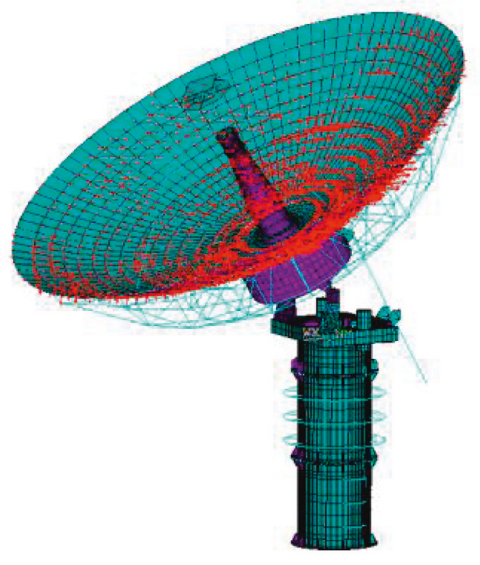

(a)

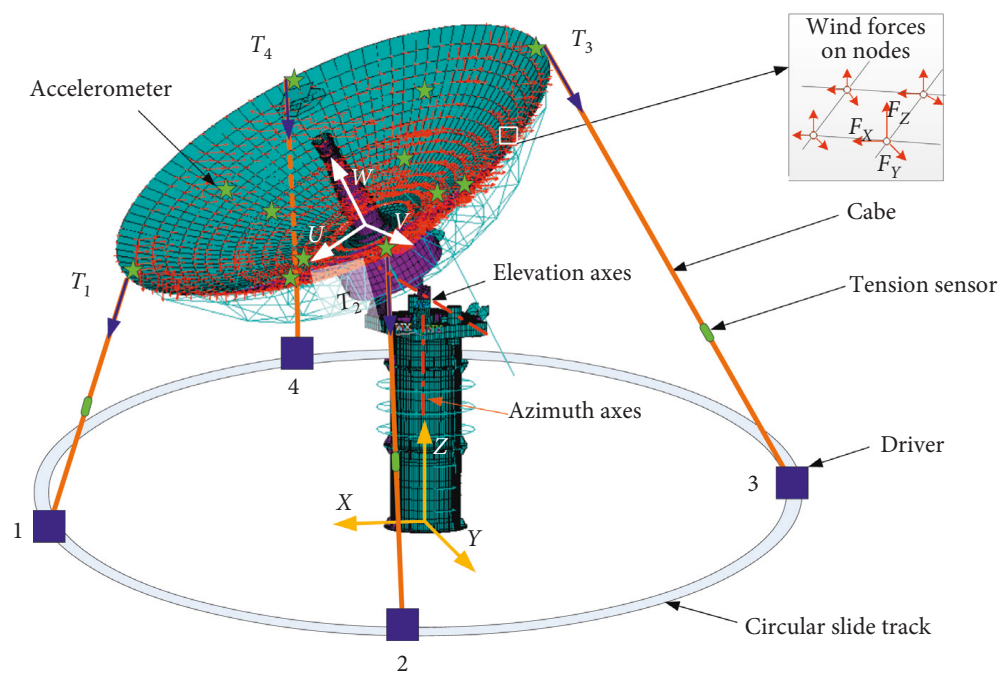

(b)

FIGURE 1: Schematic diagram of a large antenna under wind disturbance. (a) Original structure. (b) Original structure equipped with the adaptive system.

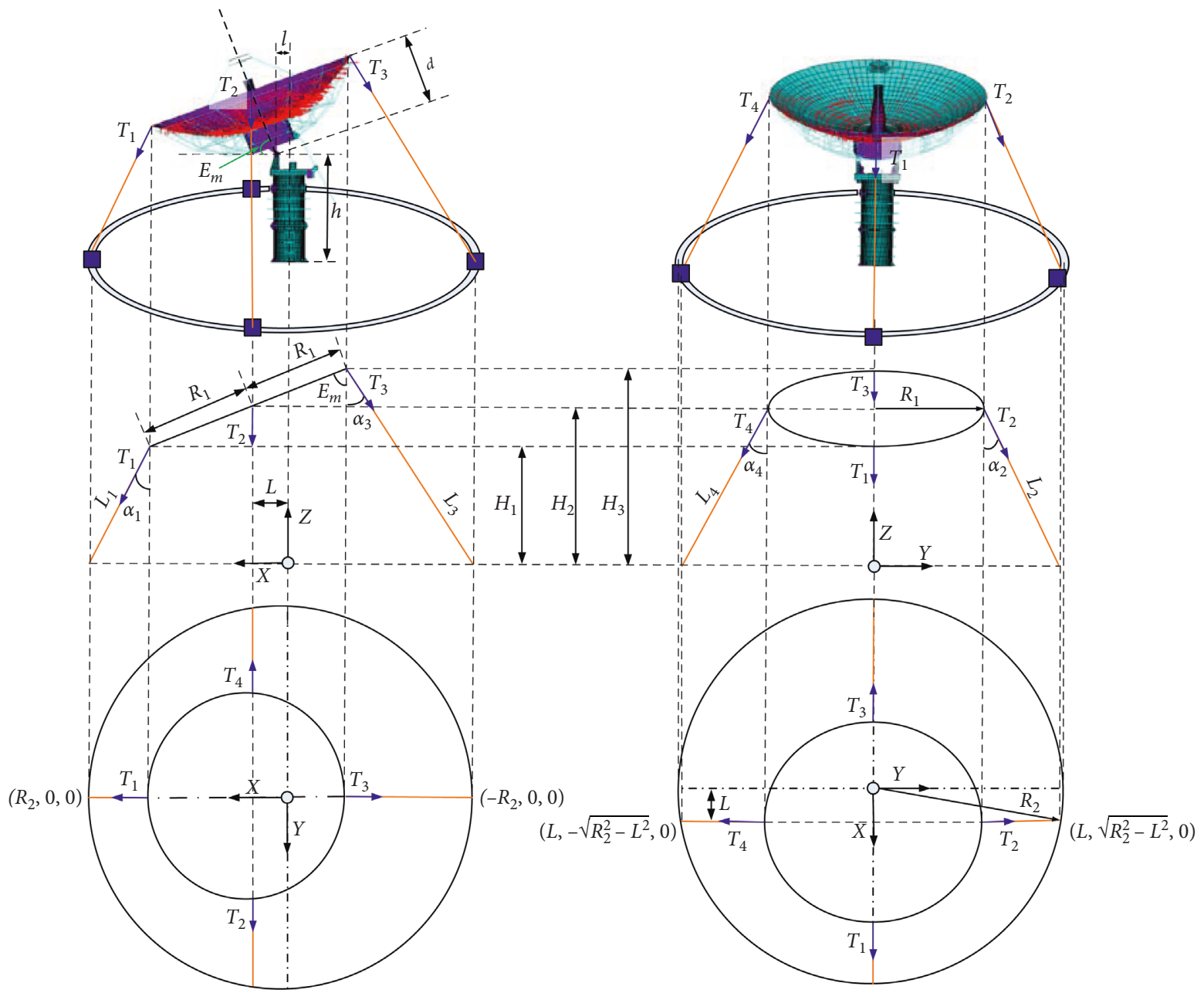

Figure 2: Geometric relations of various elements. 


\section{A Low-Order Flexible Antenna Model under Transient Wind Forces and Cable Tensions in a Three-Dimensional Space}

A flexible antenna structural model consisting of numerous nodes and elements has hundreds of thousands of degrees of freedom. The model can be characterized by matrices representing the mass, stiffness, and damping of each component. The inputs of the flexible model are transient wind forces and cable tensions in a three-dimensional space. The outputs of the model are accelerations of measurement points for obtaining the state of the vibration in Section 4 and displacements of all reflector nodes for assessing the SVD and PE in Section 6.

For describing the flexible vibration of the antenna reflector, consider the continuous-time dynamic model under moving forces in a three-dimensional space:

$$
\begin{gathered}
\mathbf{M}\left[\begin{array}{c}
\ddot{\mathbf{p}}_{x} \\
\ddot{\mathbf{p}}_{y} \\
\ddot{\mathbf{p}}_{z}
\end{array}\right]+\mathbf{D}\left[\begin{array}{l}
\dot{\mathbf{p}}_{x} \\
\dot{\mathbf{p}}_{y} \\
\dot{\mathbf{p}}_{z}
\end{array}\right]+\mathbf{K}\left[\begin{array}{l}
\mathbf{p}_{x} \\
\mathbf{p}_{y} \\
\mathbf{p}_{z}
\end{array}\right]=\left[\begin{array}{l}
\mathbf{B}_{x} \\
{[0]} \\
{[0]}
\end{array}\right]\left(\mathbf{F}_{x}+\mathbf{B}_{T x}\left[\begin{array}{c}
T_{1} \\
T_{2} \\
T_{3} \\
T_{4}
\end{array}\right]\right) \\
\left.+\left[\begin{array}{c}
{[0]} \\
\mathbf{B}_{y} \\
{[0]}
\end{array}\right]\left(\begin{array}{l}
T_{1} \\
T_{2} \\
T_{3} \\
T_{4}
\end{array}\right]\right)+\left[\begin{array}{l}
{[0]} \\
{[0]} \\
\mathbf{F}_{y}+\mathbf{B}_{z}
\end{array}\right]\left(\mathbf{F}_{z}+\mathbf{B}_{T z}\left[\begin{array}{c}
T_{1} \\
T_{2} \\
T_{3} \\
T_{4}
\end{array}\right]\right) \\
{\left[\begin{array}{l}
\mathbf{a}_{x} \\
\mathbf{a}_{y} \\
\mathbf{a}_{z}
\end{array}\right]=\mathbf{C}_{0}\left[\begin{array}{l}
\ddot{\mathbf{p}}_{x} \\
\ddot{\mathbf{p}}_{y} \\
\ddot{\mathbf{p}}_{z}
\end{array}\right],} \\
{\left[\begin{array}{l}
\mathbf{d}_{x} \\
\mathbf{d}_{y} \\
\mathbf{d}_{z}
\end{array}\right]=\left[\begin{array}{l}
\mathbf{p}_{x} \\
\mathbf{p}_{y} \\
\mathbf{p}_{z}
\end{array}\right]}
\end{gathered}
$$

Here, the antenna reflector has $t$ nodes, and the number of measuring points is $m . \mathbf{p}=\left[\mathbf{p}_{x}^{T} \mathbf{p}_{y}^{T} \mathbf{p}_{z}^{T}\right]^{T}$, $\dot{\mathbf{p}}=\left[\dot{\mathbf{p}}_{x}^{T} \dot{\mathbf{p}}_{y}^{T} \dot{\mathbf{p}}_{z}^{T}\right]^{T}$, and $\ddot{\mathbf{p}}=\left[\ddot{\mathbf{p}}_{x}^{T} \ddot{\mathbf{p}}_{y}^{T} \ddot{\mathbf{p}}_{z}^{T}\right]^{T}$ are the $3 t \times 1$ nodal displacement, velocity, and acceleration vectors of the reflector in the $X Y Z$ coordinate system. Also, $\mathbf{F}_{x}, \mathbf{F}_{y}$, and $\mathbf{F}_{z}$ are all the $t \times 1$ input vectors of wind forces which can act on any node of the reflector in $X, Y$, and $Z$ directions, respectively, as shown in Figure 1; the $4 \times 1$ input vector of cable tensions is $\mathbf{T}=\left[T_{1} T_{2} T_{3} T_{4}\right]^{T} ; \mathbf{B}_{T x}, \mathbf{B}_{T y}$, and $\mathbf{B}_{T z}$ are the tension decomposition matrices which depend on equations (4)-(7). $\mathbf{a}=\left[\mathbf{a}_{x}^{T} \mathbf{a}_{y}^{T} \mathbf{a}_{z}^{T}\right]^{T}$ and $\mathbf{d}=\left[\mathbf{d}_{x}^{T} \mathbf{d}_{y}^{T} \mathbf{d}_{z}^{T}\right]^{T}$ are the $3 m \times 1$ output vector of measuring points and $3 t \times 1$ output vector of nodes. And the $3 t \times 3 t$ matrices $\mathbf{M}, \mathbf{D}$, and $\mathbf{K}$ are for the masses, damping factors, and stiffness parameters, respectively. The input matrices $\mathbf{B}_{x}, \mathbf{B}_{y}$, and $\mathbf{B}_{z}$ all have the dimension $t \times t$. The output matrix $\mathbf{C}_{0}$ has the dimension $3 m \times 3 t$.

For getting a real-time measurement model, a low-order antenna model based on the modal superposition method is introduced. We choose the $n$ order modes. To express the structure in modal coordinates, the $n \times 1$ modal displacement $\mathbf{p}_{m}$ is introduced, which satisfies

$$
\mathbf{p}=\Phi \mathbf{p}_{m}
$$

Here, the $3 t \times n$ matrix of mode shapes $\Phi$ in $X, Y$, and $Z$ directions is defined as

$$
\boldsymbol{\Phi}=\left[\begin{array}{c}
\Phi_{x} \\
\Phi_{y} \\
\boldsymbol{\Phi}_{z}
\end{array}\right]=\left[\begin{array}{ccc}
\varphi_{x_{-} 11} & \ldots & \varphi_{x_{-} 1 \mathrm{n}} \\
\ldots & \ldots & \ldots \\
\varphi_{x_{-} t 1} & \ldots & \varphi_{x_{-} t n} \\
\varphi_{y_{-} 11} & \ldots & \varphi_{y_{-} 1 \mathrm{n}} \\
\ldots & \ldots & \ldots \\
\varphi_{y_{-} t 1} & \ldots & \varphi_{y_{-} t n} \\
\varphi_{z_{-} 11} & \ldots & \varphi_{z_{-} 1 \mathrm{n}} \\
\ldots & \ldots & \ldots \\
\varphi_{z_{-} t 1} & \ldots & \varphi_{z_{-} t n}
\end{array}\right],
$$

where $\varphi_{x_{-} i j}$ is the $i$ th node modal displacement of the $j$ th mode in the $X$ direction.

Substituting (11) into (8) and (9), left multiplying (8) by $\boldsymbol{\Phi}^{T}$, and assuming proportional damping, we obtain

$$
\begin{aligned}
\mathbf{M}_{m} \ddot{\mathbf{p}}_{m}+\mathbf{D}_{m} \dot{\mathbf{p}}_{m}+\mathbf{K}_{m} \mathbf{p}_{m}= & \boldsymbol{\Phi}_{x}^{T} \mathbf{B}_{x}\left(\mathbf{F}_{x}+\mathbf{B}_{T x} \mathbf{T}\right) \\
& +\boldsymbol{\Phi}_{y}^{T} \mathbf{B}_{y}\left(\mathbf{F}_{y}+\mathbf{B}_{T y} \mathbf{T}\right) \\
& +\boldsymbol{\Phi}_{z}^{T} \mathbf{B}_{z}\left(\mathbf{F}_{z}+\mathbf{B}_{T z} \mathbf{T}\right), \\
\mathbf{a}= & \mathbf{C}_{0} \boldsymbol{\Phi} \ddot{\mathbf{p}}_{m} \\
\mathbf{d}= & \boldsymbol{\Phi} \mathbf{p}_{m} .
\end{aligned}
$$

In this expression, now the modal mass matrix $\mathbf{M}_{m}$, modal stiffness matrix $\mathbf{K}_{m}$, and modal damping matrix $\mathbf{D}_{m}$ satisfy

$$
\begin{aligned}
\mathbf{M}_{m} & =\boldsymbol{\Phi}^{T} \mathbf{M} \boldsymbol{\Phi}, \\
\mathbf{K}_{m} & =\boldsymbol{\Phi}^{T} \mathbf{K} \boldsymbol{\Phi} \\
\mathbf{D}_{m} & =\boldsymbol{\Phi}^{T} \mathbf{D} \boldsymbol{\Phi} .
\end{aligned}
$$

Next, left multiplying (12) by $\mathbf{M}_{m}^{-1}$ yields

$$
\begin{gathered}
\ddot{\mathbf{p}}_{m}+\Gamma \dot{\mathbf{p}}_{m}+\Lambda \mathbf{p}_{m}=\mathbf{B}_{i} \mathbf{F}, \\
\mathbf{B}_{i}=\left[\mathbf{M}_{m}^{-1} \boldsymbol{\Phi}_{x}^{T} \mathbf{B}_{x}, \mathbf{M}_{m}^{-1} \boldsymbol{\Phi}_{y}^{T} \mathbf{B}_{y}, \mathbf{M}_{m}^{-1} \boldsymbol{\Phi}_{z}^{T} \mathbf{B}_{z}\right], \\
\mathbf{F}=\left[\begin{array}{c}
\mathbf{F}_{x}+\mathbf{B}_{T x} \mathbf{T} \\
\mathbf{F}_{y}+\mathbf{B}_{T y} \mathbf{T} \\
\mathbf{F}_{z}+\mathbf{B}_{T z} \mathbf{T}
\end{array}\right], \\
\Gamma=\mathbf{M}_{m}^{-1} \mathbf{D}_{m}=\operatorname{diag}\left(2 \xi_{1} \omega_{1}, 2 \xi_{2} \omega_{2}, \ldots, 2 \xi_{n} \omega_{n}\right), \\
\boldsymbol{\Lambda}=\mathbf{M}_{m}^{-1} \mathbf{K}_{m}=\operatorname{diag}\left(\omega_{1}^{2}, \omega_{2}^{2}, \ldots, \omega_{n}^{2}\right),
\end{gathered}
$$

where $\xi_{i}$ is the damping ratio of the $i$ th mode and $\omega_{i}$ is the natural frequency of the $i$ th mode.

For the sake of convenience, (15) and (13) can be transformed into state-space equations: 


$$
\begin{aligned}
& \dot{\mathbf{x}}(t)=\mathbf{A}_{c} \mathbf{x}(t)+\mathbf{B}_{c} \mathbf{F}(t) \\
& \mathbf{a}(t)=\mathbf{C}_{a} \mathbf{x}(t)+\mathbf{D}_{c} \mathbf{F}(t), \\
& \mathbf{d}=\mathbf{C}_{d} \mathbf{x}(t), \\
& \mathbf{A}_{c}=\left[\begin{array}{cc}
{[0]} & \mathbf{I} \\
-\boldsymbol{\Lambda} & -\Gamma
\end{array}\right], \\
& \mathbf{B}_{c}=\left[\begin{array}{c}
{[0]} \\
\mathbf{B}_{i}
\end{array}\right] \text {, } \\
& \mathrm{C}_{a}=\left[-\mathrm{C}_{o} \boldsymbol{\Phi} \Lambda-\mathrm{C}_{o} \Phi \Gamma\right], \\
& \mathbf{C}_{d}=\left[\begin{array}{ll}
\boldsymbol{\Phi} & {[0]}
\end{array}\right] \text {, } \\
& \mathbf{D}_{c}=\mathbf{C}_{o} \Phi \mathbf{B}_{i} \text {, } \\
& \mathbf{x}(t)=\left[\begin{array}{c}
\mathbf{p}_{m}(t) \\
\dot{\mathbf{p}}_{m}(t)
\end{array}\right] .
\end{aligned}
$$

\section{Real-Time Estimation of the Vibration State by Acceleration Measurements and the Low-Order Flexible Model considering Equivalent Identification of Force}

First, by discretizing the continuous system of (18) and (19) via a zero-order holder, we obtain

$$
\begin{aligned}
\mathbf{x}_{k+1} & =\mathbf{A} x_{k}+\mathbf{B} \mathbf{F}_{k}+\mathbf{w}_{k}, \\
\mathbf{a}_{k} & =\mathbf{C}_{a} \mathbf{x}_{k}+\mathbf{D}_{c} \mathbf{F}_{k}+\mathbf{v}_{k}, \\
\mathbf{d}_{k} & =\mathbf{C}_{d} \mathbf{x}_{k},
\end{aligned}
$$

where $\mathbf{A}=e^{\mathbf{A}_{c} \Delta t}$, in which $\Delta t$ is the sampling time; $\mathbf{B}=(\mathbf{A}-\mathbf{I}) \mathbf{A}_{c}^{-1} \mathbf{B}_{c} ; \mathbf{w}_{k}$ is the model error caused by model reduction, and its covariance matrices $\mathbf{Q}=\mathbb{E}\left(\mathbf{w}_{k} \mathbf{w}_{l}^{T}\right) \geq 0 ; \mathbf{v}_{k}$ is the measurement noise, and its covariance matrices $\mathbf{R}=\mathbb{E}\left(\mathbf{v}_{k} \mathbf{v}_{l}^{T}\right) \geq 0$.

However, $\mathbf{F}_{k}$ and $\mathbf{x}_{k}$ are unknown in real, so $\mathbf{F}_{k}$ and $\mathbf{x}_{k}$ should be identified successively. Gillijns presented an unbiased minimum-variance input and state estimation method for linear discrete-time systems which can solve this problem [14]. The method has been used for input-response estimation by acceleration measurements for structural systems [15, 16]. However, these papers only loaded and identified one or two node forces in one direction. Identifying multisource moving force in a three-dimensional space not only leads to poor computation efficiency but also does not satisfy the direct invertibility requirement in which the number of forces to be identified cannot exceed the number of modes [17]. Since our goal is to estimate $\mathbf{x}_{k}$, it is not necessary to identify the actual multisource moving forces (wind forces and cable tensions) in a three-dimensional space. Therefore, under the premise of stability, observability, controllability, and direct invertibility criteria, the complex problem of identifying unknown spatial distribution forces can be simplified into identifying the equivalent forces of some nodes in a certain direction.

The state forces $\mathbf{F}_{k}^{x}$ are the same before and after equivalent:

$$
\mathbf{B F}_{k}=\mathbf{B}_{e 1} \mathbf{F}_{k}^{\prime}=\mathbf{F}_{k}^{x}
$$

For the estimator design, the new state equation of (23) is

$$
\begin{aligned}
\mathbf{x}_{k+1} & =\mathbf{A} x_{k}+\mathbf{B F}_{k}+\mathbf{w}_{k}=\mathbf{A} \mathbf{x}_{k}+\mathbf{B}_{e 1} \mathbf{F}_{k}^{\prime}+\mathbf{w}_{k}, \\
\mathbf{B}_{e 1} & =\mathbf{B}_{\square} \mathbf{B}_{F}, \\
\mathbf{B} & =\left[\mathbf{B}_{X}, \mathbf{B}_{Y}, \mathbf{B}_{Z}\right], \\
\mathbf{B}_{F} & =\left[\begin{array}{cccc}
B_{\square 11} & B_{\square 12} & \ldots & B_{\square 1 q} \\
B_{\square 21} & B_{\square 22} & \ldots & B_{\square 2 q} \\
\ldots & \ldots & \ldots & \ldots \\
B_{\square t 1} & B_{\square t 1} & \ldots & B_{\square t q}
\end{array}\right],
\end{aligned}
$$

where $\square$ can be a certain direction: $X, Y$, or $Z$, and $\mathbf{B}_{\square} \in\left\{\mathbf{B}_{X}, \mathbf{B}_{Y}, \mathbf{B}_{Z}\right\} . \mathbf{B}_{F}$ is the input matrix of force positions. If the $j$ th identified equivalent force is in the $X$ direction at the $i$ th node, then $B_{X i j}=1$ in $\mathbf{B}_{F}$ or otherwise $B_{X i j}=0$.

The multisource moving forces in a three-dimensional space are

$$
\mathbf{F}_{k}=\left[\begin{array}{llllllllllll}
\mathbf{F}_{k}^{1 X} & \mathbf{F}_{k}^{2 X} & \ldots & \mathbf{F}_{k}^{t X} & \mathbf{F}_{k}^{1 Y} & \mathbf{F}_{k}^{2 Y} & \ldots & \mathbf{F}_{k}^{t Y} & \mathbf{F}_{k}^{1 Z} & \mathbf{F}_{k}^{2 Z} & \ldots & \mathbf{F}_{k}^{t Z}
\end{array}\right]^{T},
$$

where $\mathbf{F}_{k}^{i X}, \mathbf{F}_{k}^{i Y}$, and $\mathbf{F}_{k}^{i Z}$ are the $i$ th node forces at step $k$ in $X$, $Y$, and $Z$ directions.

The theoretical equivalent forces on $q$ nodes are

$$
\mathbf{F}_{k}^{\prime}=\mathbf{B}_{e 1}^{+} \mathbf{B} \mathbf{F}_{k}
$$

where $\mathbf{B}_{e 1}^{+}$is the pseudoinverse (pinv) of $\mathbf{B}_{e 1}$. And the pinv formed by singular value decomposition not only solves the inverse of the nonsquare matrix but also avoids numerical instabilities due to rank deficiency.

The new equation of (24) is

$$
\begin{aligned}
\mathbf{a}_{k} & =\mathbf{C}_{a} \mathbf{x}_{k}+\mathbf{D}_{c} \mathbf{F}_{k}+\mathbf{v}_{k}=\mathbf{C}_{a} \mathbf{x}_{k}+\mathbf{D}_{e 1} \mathbf{F}_{k}^{\prime}+\mathbf{v}_{k}, \\
\mathbf{D}_{e 1} & =\mathbf{D}_{c} \mathbf{B}^{+} \mathbf{B}_{e 1} .
\end{aligned}
$$

Then, based on the acceleration measurement and the new system model comprising $\mathbf{A}, \mathbf{B}_{e 1}, \mathbf{C}_{a}$, and $\mathbf{D}_{e 1}$, the realtime estimation of the identified equivalent forces and vibration state $\mathbf{x}_{k \mid k}$ is obtained in an unbiased minimumvariance way; for details on the procedures, readers can refer to the original works $[14,15]$, and the equations used in the paper are given in Appendix.

\section{Cable Tension Controller to Suppress the Vibration Based on LQR}

For the controller design, the new state equation is

$$
\begin{aligned}
\mathbf{x}_{k+1} & =\mathbf{A} x_{k}+\mathbf{B} \mathbf{F}_{k}+\mathbf{w}_{k}=\mathbf{A} \mathbf{x}_{k}+\mathbf{F}_{k}^{x}+\mathbf{w}_{k}, \\
\mathbf{B}_{e 2} & =\mathbf{I} .
\end{aligned}
$$


Based on the LQR, the state force that suppresses vibration is obtained from the estimated value of the vibration state $\mathbf{x}_{k \mid k}$ :

$$
\mathbf{F}_{k}^{-x}=-\mathbf{K}_{\mathrm{LQR}} \mathbf{x}_{k \mid k} \text {. }
$$

The optimal gain $\mathbf{K}_{\mathrm{LQR}}$ is derived from minimization of the quadratic cost function:

$$
\begin{aligned}
\min : J\left(\mathbf{F}^{-x}\right) & =\sum_{k=0}^{\infty}\left(\mathbf{x}_{k \mid k}^{T} \mathbf{Q}_{1} \mathbf{x}_{k \mid k}+\left(\mathbf{F}_{k}^{-x}\right)^{T} \mathbf{R}_{1} \mathbf{F}_{k}^{-x}\right), \\
\mathbf{K}_{\mathrm{LQR}} & =\left(\mathbf{B}_{e 2}^{T} \mathbf{S B}_{e 2}+\mathbf{R}_{1}\right)^{-1} \mathbf{B}_{e 2}^{T} \mathbf{S A} .
\end{aligned}
$$

Here, the weighting parameters $\mathbf{Q}_{1}$ and $\mathbf{R}_{1}$ affected the system output and control input, respectively. Therefore, they certainly satisfied the following conditions: $\mathbf{Q}_{1} \geq 0$ and $\mathbf{R}_{1}>0$.

The infinite horizon solution $\mathbf{S}$ of the associated discretetime Riccati equation satisfies

$$
\mathbf{A}^{T} \mathbf{S A}-\mathbf{S}-\mathbf{A}^{T} \mathbf{S B}_{e 2}\left(\mathbf{B}_{e 2}^{T} \mathbf{S B}_{e 2}+\mathbf{R}_{1}\right)^{-1} \mathbf{B}_{e 2}^{T} \mathbf{S A}+\mathbf{Q}_{1}=0 .
$$

$\mathbf{F}_{k}^{-x}$ should be generated by the expected cable tension of the next time step:

$$
\mathbf{F}_{k}^{-x}=\mathbf{B}\left[\begin{array}{c}
\mathbf{B}_{T x} \mathbf{T}_{k+1}^{e} \\
\mathbf{B}_{T y} \mathbf{T}_{k+1}^{e} \\
\mathbf{B}_{T z} \mathbf{T}_{k+1}^{e}
\end{array}\right]=\mathbf{B B}_{T} \mathbf{T}_{k+1}^{e} .
$$

Then, the expected tension distribution of the cables is found by (32) and (35):

$$
\mathbf{T}_{k+1}^{e}=-\left(\mathbf{B B}_{T}\right)^{+} \mathbf{K}_{\mathrm{LQR}} \mathbf{x}_{k \mid k} .
$$

And a cable-actuator control system (CACS) can be approximately considered a first order of inertia [18]:

$$
\dot{T}_{i}=-\frac{1}{\tau} T_{i}+\frac{1}{\tau} T_{i}^{e}
$$

where $T_{i}$ and $T_{i}^{e}$ represent the actual output tension and the expected tension of the $i$ th cable. $\tau$ denotes the time constant on inertial constraint units subjected to the element in the whole closed-loop actuator system. Therefore, the servo bandwidth of the CACS is $1 / \tau$.

Then, with the sampling time $\Delta t$, by discretizing the continuous cable-actuator control system via a zero-order holder and considering the saturation constraint, we obtain

$$
\begin{aligned}
\mathbf{T}_{k+1} & =\left[\begin{array}{cccc}
S_{1} & 0 & 0 & 0 \\
0 & S_{2} & 0 & 0 \\
0 & 0 & S_{3} & 0 \\
0 & 0 & 0 & S_{4}
\end{array}\right]\left[\begin{array}{l}
T_{1 \_k+1} \\
T_{2 \_k+1} \\
T_{3_{-} k+1} \\
T_{4 \_k+1}
\end{array}\right] \\
& =\left[\begin{array}{cccc}
S_{1} & 0 & 0 & 0 \\
0 & S_{2} & 0 & 0 \\
0 & 0 & S_{3} & 0 \\
0 & 0 & 0 & S_{4}
\end{array}\right]\left[\begin{array}{l}
e^{-(1 / \tau) \Delta t} T_{1 \_k}+\left(1-e^{-(1 / \tau) \Delta t}\right) T_{1 \_k}^{e} \\
e^{-(1 / \tau) \Delta t} T_{2 \_k}+\left(1-e^{-(1 / \tau) \Delta t}\right) T_{2 \_k}^{e} \\
e^{-(1 / \tau) \Delta t} T_{3_{-} k}+\left(1-e^{-(1 / \tau) \Delta t}\right) T_{3 \_k}^{e} \\
e^{-(1 / \tau) \Delta t} T_{4 \_k}+\left(1-e^{-(1 / \tau) \Delta t}\right) T_{4 \_k}^{e}
\end{array}\right] \\
= & \mathbf{S}_{T}\left(e^{-(1 / \tau) \Delta t} \mathbf{I}_{4 \times 4} \mathbf{T}_{k}+\left(1-e^{-(1 / \tau) \Delta t}\right) \mathbf{I}_{4 \times 4} \mathbf{T}_{k}^{e}\right),
\end{aligned}
$$

where the unit matrix $\mathbf{I}_{4 \times 4}$ has dimensions of $4 \times 4$. The saturation constraint $\mathbf{S}_{T}$ used to limit output tension to the upper and lower saturation values is

$$
S_{i}= \begin{cases}1, & \text { for } T_{\max } \geq T_{i} \geq 0, \\ \frac{T_{\max }}{T_{i}}, & \text { for } T_{i}>T_{\max }, \\ 0, & \text { otherwise, }\end{cases}
$$

where $T_{\max }$ cannot exceed the allowable stress of the antenna structure and the cable.

\section{Simulation Examples}

The transient wind disturbance rejection simulation of a $7.3 \mathrm{~m}$ antenna is used to illustrate the adaptive system. Table 1 shows the parameters used for simulation under the largest windward area. The material of the rope is nylon whose Young's modulus is denoted by $E$, and the crosssectional area with a diameter of $1.8 \mathrm{~cm}$ is denoted by $A_{r}$ [19]. $T_{\max }$ is set to $1000 \mathrm{~N}$. To ensure real-time monitoring and control, the runtime of each recursive step in the adaptive system should be smaller than the sampling time. We use MATLAB commands "tic" and "toc" to test each recursive step runtime. In an ordinary computer (with the Intel Core i5-6500 CPU and 16 GB RAM), each recursive time step takes about $0.7 \mathrm{~ms}$. So the sampling time is set to $1 \mathrm{~ms}$ which is larger than $0.7 \mathrm{~ms}$.

6.1. Transient Wind Model of a $7.3 \mathrm{~m}$ Antenna. Wind load can be classified into mean wind and gust wind. The wind speed at any point can be expressed as the sum of mean wind speed and gust wind speed $[20,21]$ :

$$
v=v_{Z b}\left(\frac{Z}{Z_{b}}\right)^{\alpha_{s}}+\Delta v,
$$


TABLe 1: Parameters used for simulation.

\begin{tabular}{lc}
\hline & Value \\
\hline Parameters of the adaptive system & 0 \\
$E_{m}\left(^{\circ}\right)$ & 3.8915 \\
$h(\mathrm{~m})$ & 2.4445 \\
$d(\mathrm{~m})$ & 0.4150 \\
$l(\mathrm{~m})$ & 3.65 \\
$R_{1}(\mathrm{~m})$ & 10 \\
$R_{2}(\mathrm{~m})$ & 2.5 \\
$E(\mathrm{Gpa})$ & $2.54 \times 10^{-4}$ \\
$A_{r}\left(\mathrm{~m}^{2}\right)$ & 2.8595 \\
$L(\mathrm{~m})$ & 0.2415 \\
$H_{1}(\mathrm{~m})$ & 3.8915 \\
$H_{2}(\mathrm{~m})$ & 7.5415 \\
$H_{3}(\mathrm{~m})$ & 88.0629 \\
$\alpha_{1}\left({ }^{\circ}\right)$ & 56.7364 \\
$\alpha_{2}\left({ }^{\circ}\right)$ & 59.6104 \\
$\alpha_{3}\left({ }^{\circ}\right)$ & 56.7364 \\
$\alpha_{4}\left({ }^{\circ}\right)$ & \\
\hline Other parameters & $v$ \\
Wind speed $(\mathrm{m} / \mathrm{s})$ & \\
Wind direction & \\
Sampling time $(\mathrm{s})$ & Front of the antenna \\
Simulation time $(\mathrm{s})$ & 0.001 \\
\hline
\end{tabular}

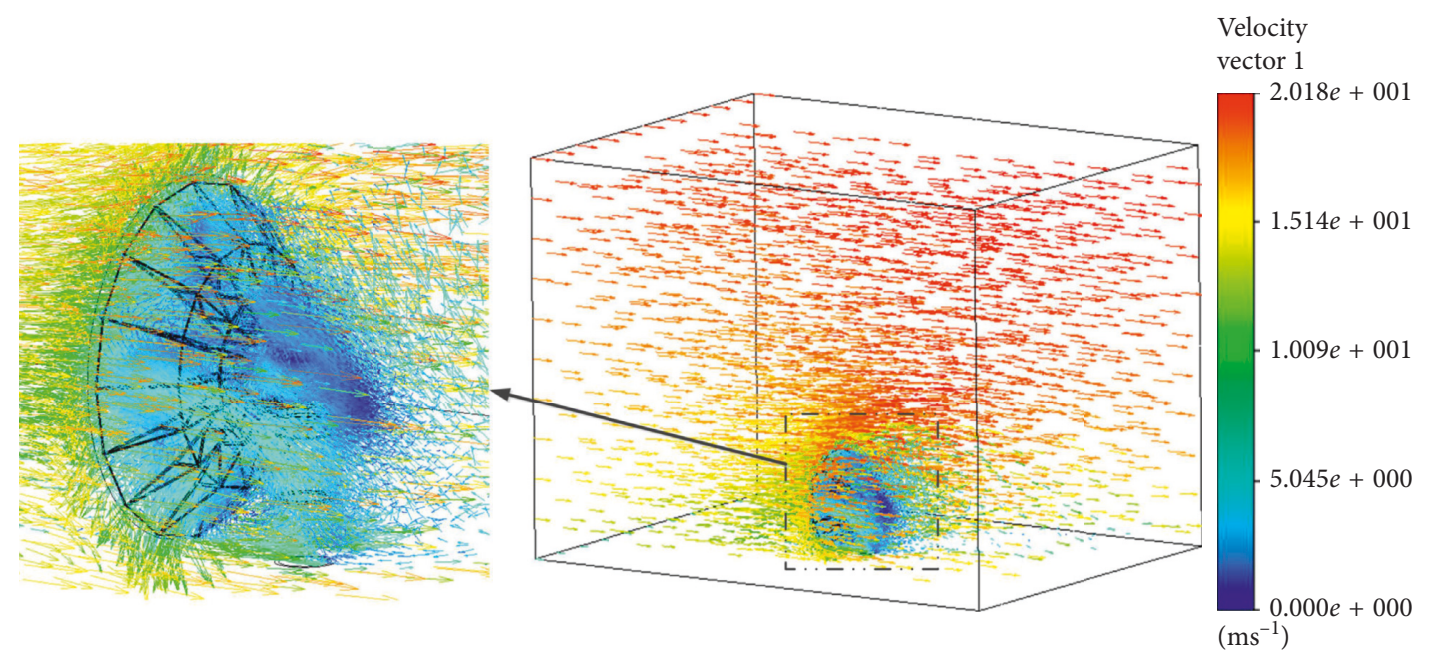

Figure 3: Wind field of the antenna.

where mean wind satisfies the exponential law relation of the wind profile, $v_{Z b}$ is the average wind speed at the reference height of $Z_{b}$ in the $X Y Z$ coordinate system and set to $10 \mathrm{~m} / \mathrm{s}$, $Z_{b}$ is set to $1 \mathrm{~m}$, and $\alpha_{s}$ is the ground roughness set to 0.16 for open terrain. And $\Delta v$ can be obtained by the Davenport filter from white noise with an average value of zero.

We set up the wind field of the antenna in the CFX software, import the wind speed data, and perform transient analysis, as shown in Figure 3. After the analysis, the transient wind forces can be obtained.

6.2. A Low-Order Flexible Antenna Model for Estimator and Controller Design. In order to get an approximate fullorder model (AFM) for real-time monitoring and control simulation in the next section and get the low-order flexible model for estimator and controller design, the modal superposition method solved by MATLAB is introduced as described in Section 3. Since the characteristics of the wind spectrum are mainly associated with low frequency, resulting in the PE in low frequency [7], we choose the AFM consisting of the first 26 modes within $50 \mathrm{~Hz}$. By loading the same wind forces, setting the same sampling rate and simulation time, the displacements of the reflector surface are obtained by transient analysis of the full-order model using ANSYS and the first 26-order antenna model with MATLAB. Then, according to the best-fitting paraboloid theory [10, 22], we get the PE of the reflector surface in the UVW coordinate system: 


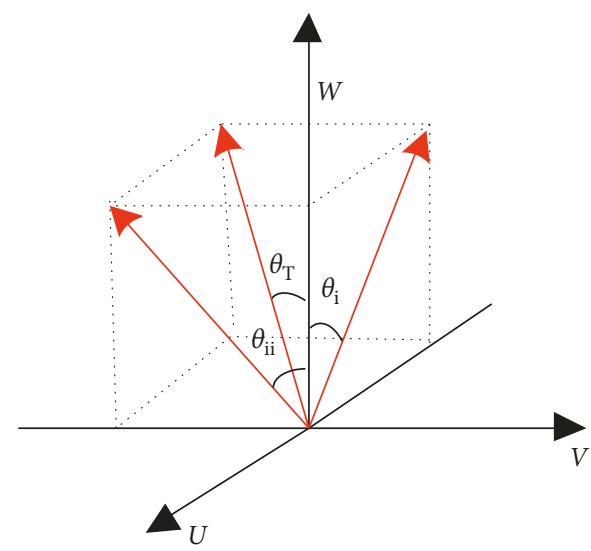

Figure 4: PEs in the UVW coordinate system.

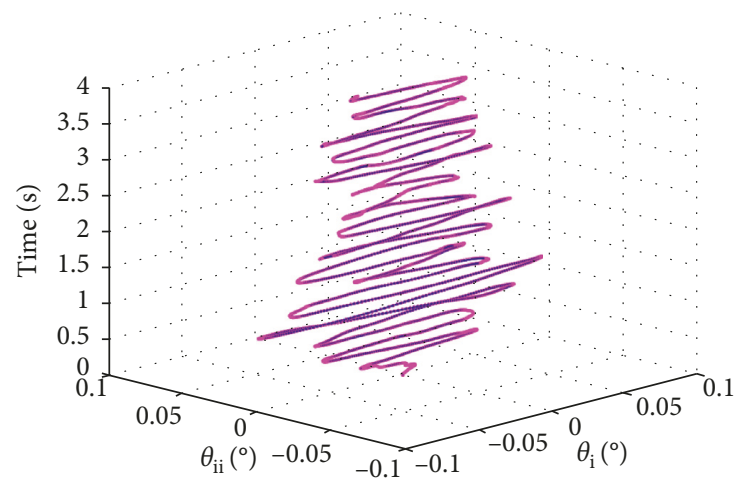

Full-order antenna model

— First 26-order antenna model

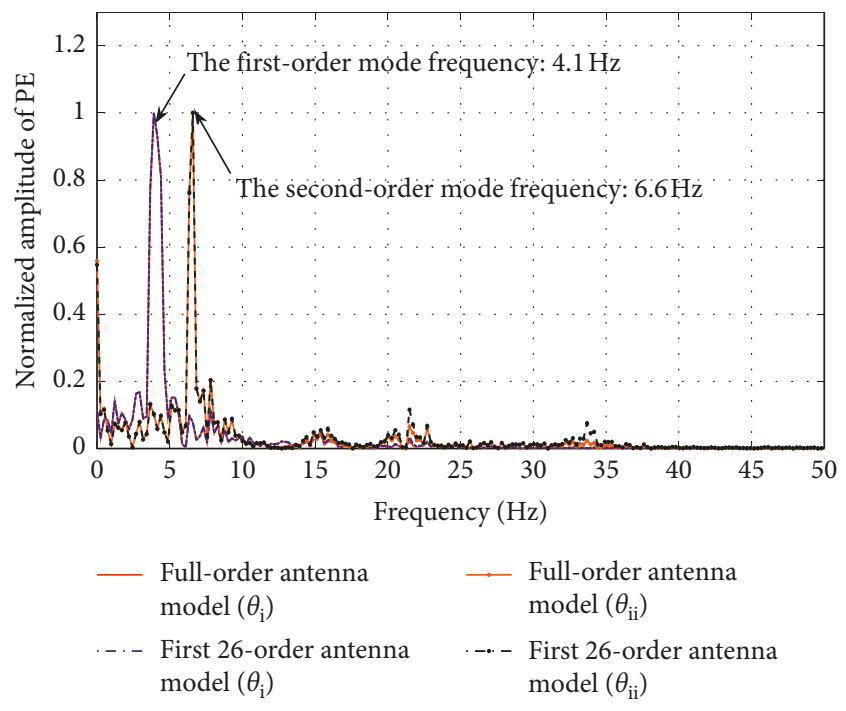

(b)

FIgURE 5: PEs under wind forces in the (a) time domain and (b) frequency domain.

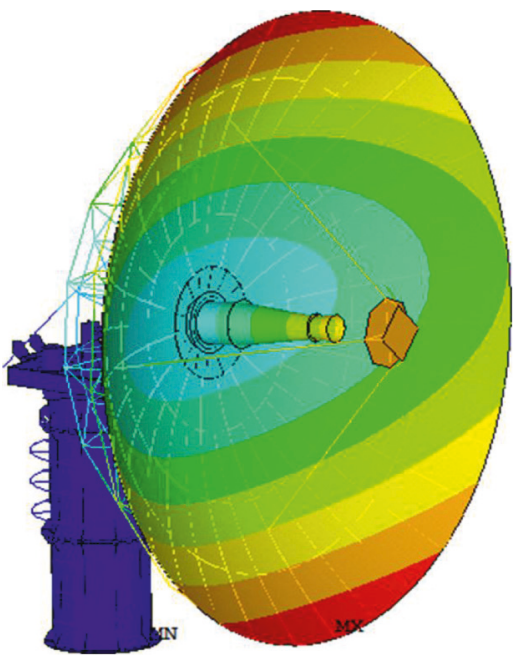

(a)

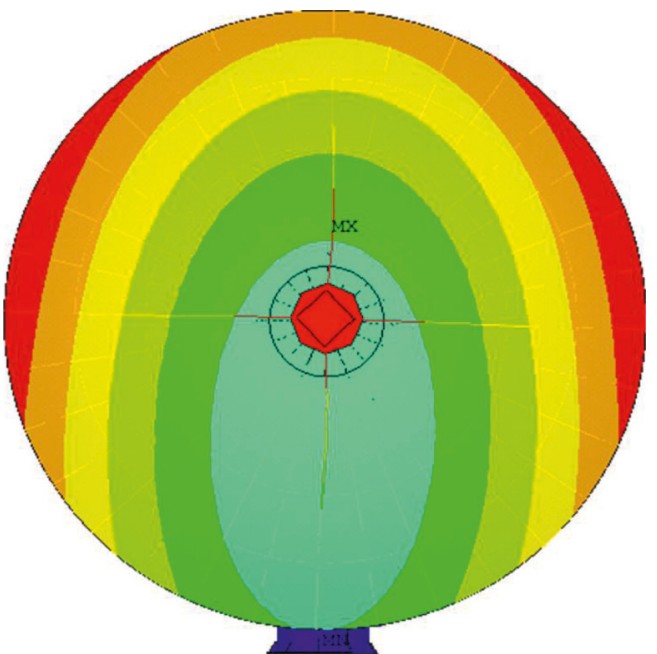

(b)

Figure 6: The first two modes of the antenna in the (a) elevation direction and (b) azimuth direction. 


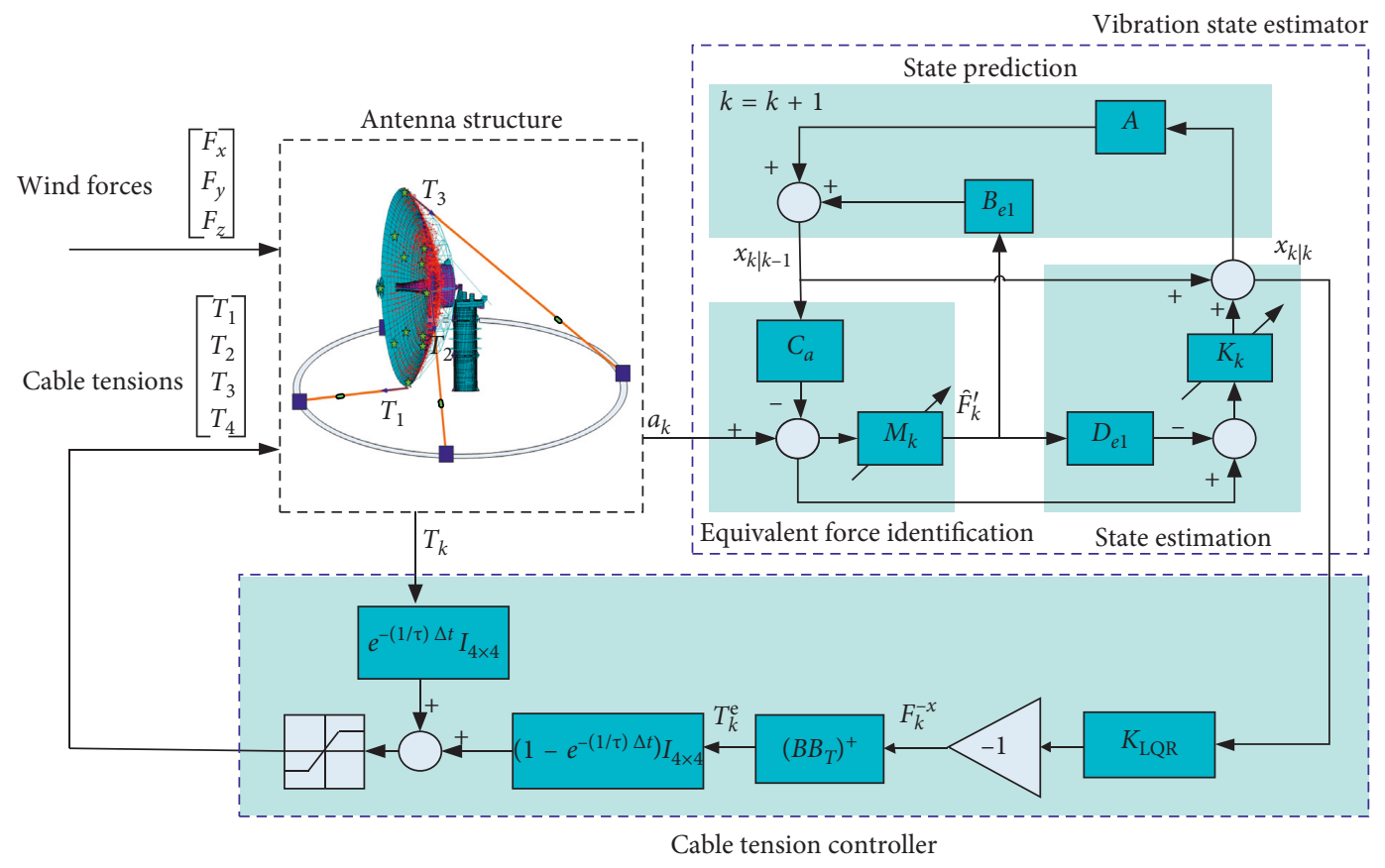

FIgURE 7: Adaptive system to suppress transient wind disturbance.

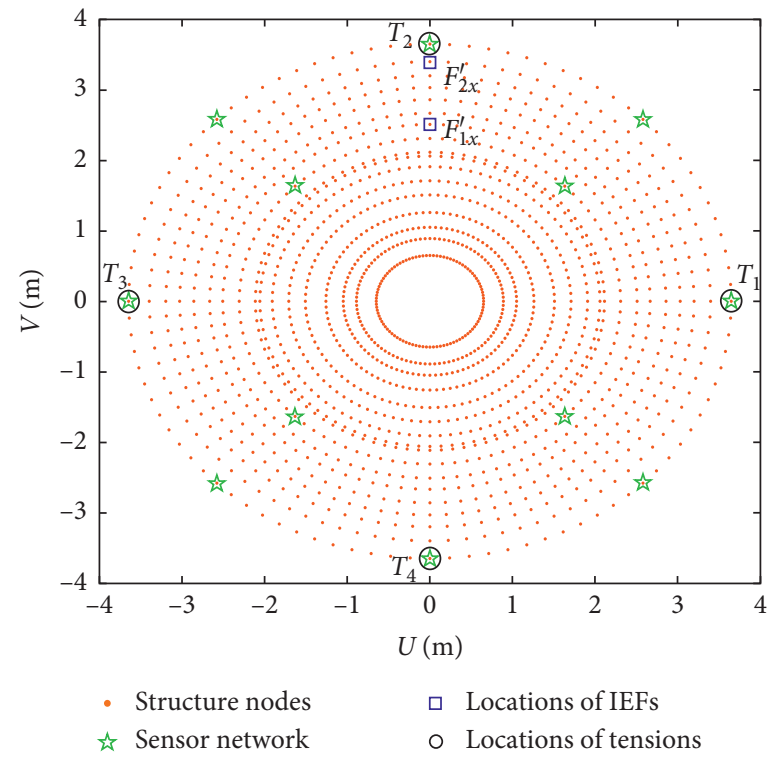

Figure 8: A network of structure nodes, sensors, and action points of IEFs and tensions.

$$
\boldsymbol{\theta}=\left[\theta_{i}, \theta_{i i}\right]^{T}=f\left(\mathbf{d}_{W}\right),
$$

where $\theta_{i}$ and $\theta_{i i}$ are the PEs in the $U W$ plane and $V W$ plane, respectively, as shown in Figure 4. And if the reflector does not have deformation, then $\theta_{T}=0 . \mathbf{d}_{W}$ obtained through coordinate transformation is the displacement vector of $t$ nodes in the $W$ direction.

The amplitude of $\boldsymbol{\theta}$ is

$$
\theta_{T}=\sqrt{\theta_{i}^{2}+\theta_{i i}^{2}}
$$

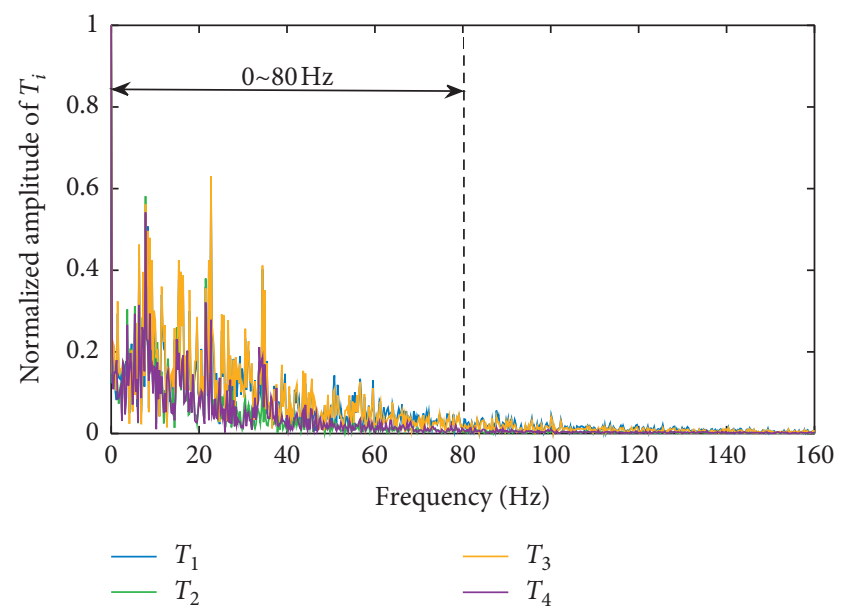

FIGURE 9: FFT of $T_{i}$ without considering the inertial constraint in the CACS.

As shown in Figure 5, the PE of the first 26 modes is basically the same as the PE of the full-order antenna model, with a relative error of $0.79 \%$. Therefore, the AFM can be used to approximate the full-order antenna model. Moreover, transient wind mainly excites the fundamental frequency mode of the elevation direction and the azimuth direction for the antenna, as shown in Figures 5 and 6. As a result, the first 2-mode antenna model within $10 \mathrm{~Hz}$ is used as the low-order flexible model.

6.3. Adaptive System to Suppress Transient Wind Disturbance. As shown in Figure 7, the antenna structure consists of the original antenna (the AFM is used in simulation) and the 


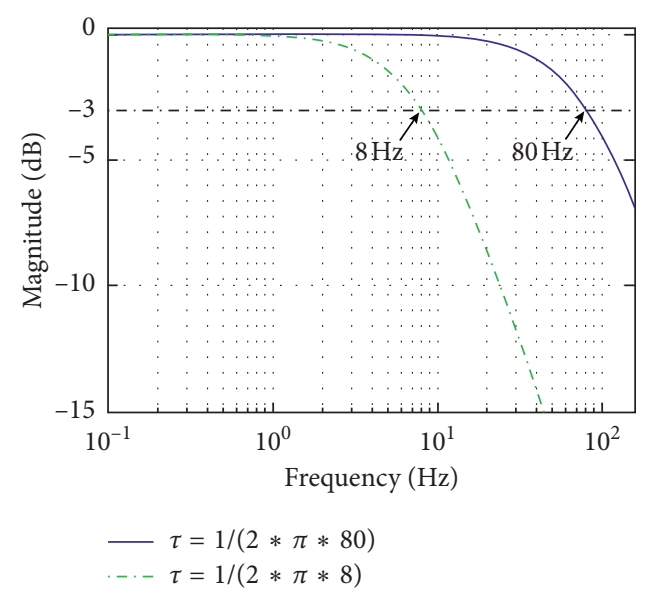

(a)

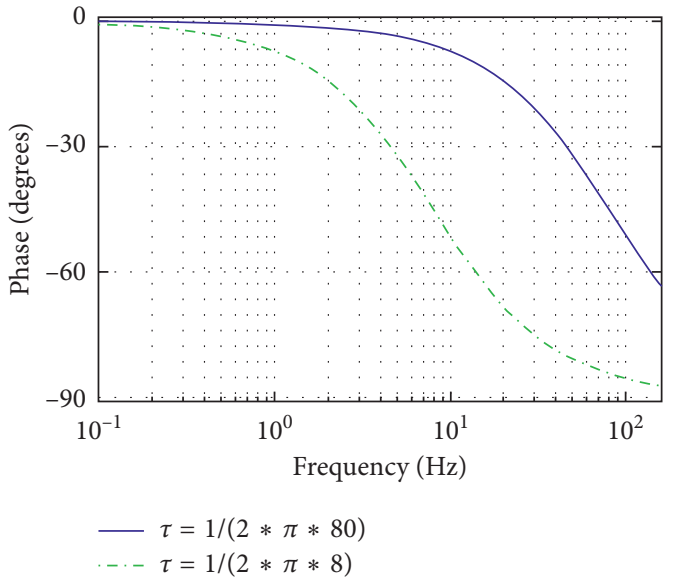

(b)

FIgure 10: Bode diagram of the CACS under two different $\tau$.

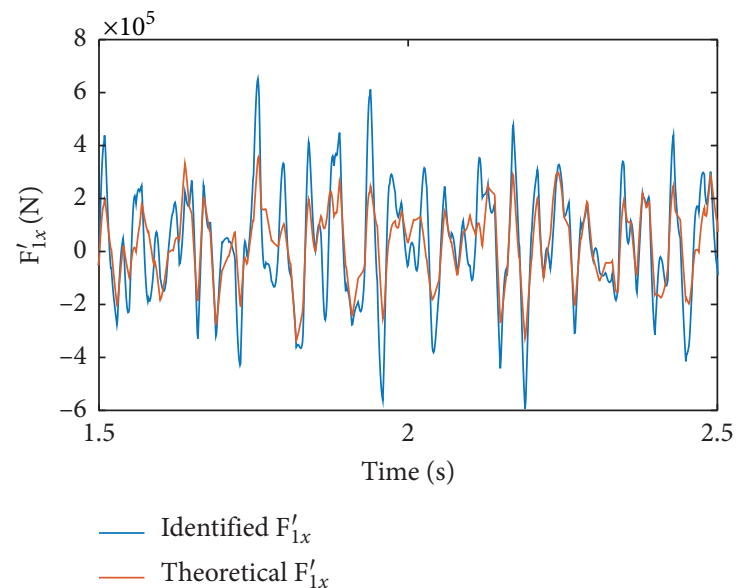

(a)

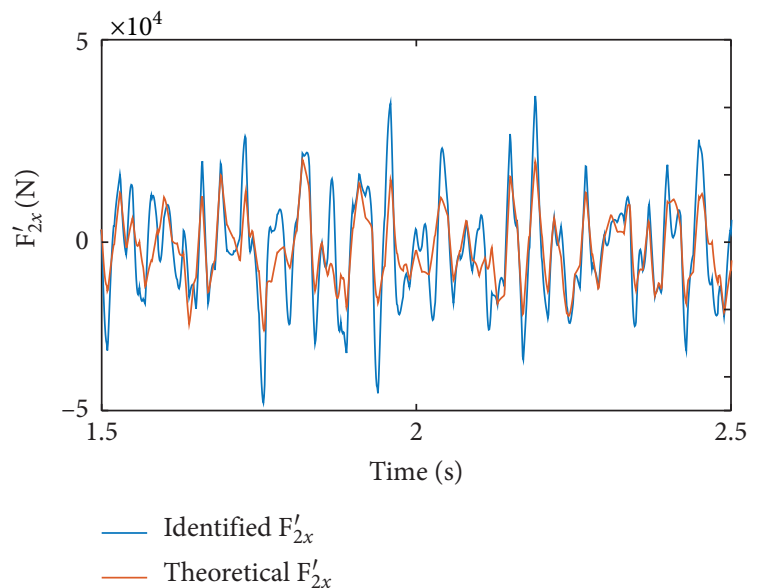

(b)

Figure 11: Equivalent forces $F_{1 x}^{\prime}(\mathrm{a})$ and $F_{2 x}^{\prime}$ (b) under $\tau=1 /(160 \pi)$.

four-cable-actuator mechanism with a circular slide track. For the calculation formula of $\mathbf{M}_{k}$ and $\mathbf{K}_{k}$, see Appendix. The initial state of the system without considering the effects of temperature and gravity is assumed to be zero, and the covariance matrices $\mathbf{Q}$ and $\mathbf{R}$ are assigned values $1 e^{-10}$ and $1 e^{-6}$ on the diagonal, respectively. The data of acceleration measurements are polluted by white noise with an average value of zero and a maximum amplitude of $1.2 \times 10^{-3} \mathrm{~m} / \mathrm{s}^{2}$.

A network of structure nodes, sensors, and action points of identified equivalent forces (IEFs) and tensions is shown in Figure 8 . The sensor placement issue is not discussed here (refer to $[20,23]$ for sensor placement strategies). For the choice of equivalent force positions, results remain sensitive to correct assumptions on the force locations [24]; they should be determined according to the actual identification effect.

For the value of $\tau$, the servo bandwidth of the CACS should be at least larger than the main frequency of structural vibration. Hence, a value of $\tau$ is set to $1 /(16 \pi)$; the servo bandwidth $(8 \mathrm{~Hz})$ corresponding to the $\tau$ is higher than the second mode frequency $(6.6 \mathrm{~Hz})$. And we think that if the servo bandwidth can cover the frequency range of larger amplitude in $T_{i}$ under the absence of inertial constraint in the CACS, the better control effect will be achieved. Therefore, after the adaptive system is operated without considering the inertial constraint in the CACS, the spectral characteristics of the ideal $T_{i}$ can be obtained, as shown in Figure 9. Since the amplitude spectrum of the ideal $T_{i}$ is mainly distributed within $80 \mathrm{~Hz}$, another value of $\tau$ is set to $1 /(160 \pi)$. Figure 10 shows the Bode diagram of the CACS under two different $\tau$; the frequency corresponding to $-3 \mathrm{~dB}$ is the servo bandwidth of the CACS.

As shown in Figure 11, the IEFs of the two nodes in the $X$ direction under $\tau=1 /(160 \pi)$ are in close agreement with the theoretical equivalent forces which are solved by (29). Similar good recognition results are also obtained under the absence of inertial constraint and $\tau=1 /(16 \pi)$.

To suppress the SVD under the transient wind disturbance, tensions of the cables can be adaptively controlled, as shown in Figure 12. With the higher servo bandwidth of the CACS under $\tau=1 /(160 \pi)$, the $T_{i}$ is very 


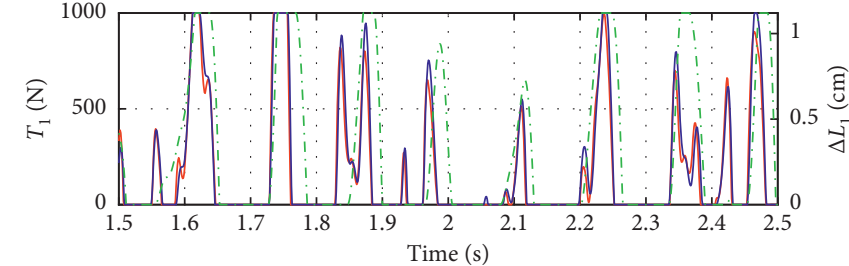

(a)

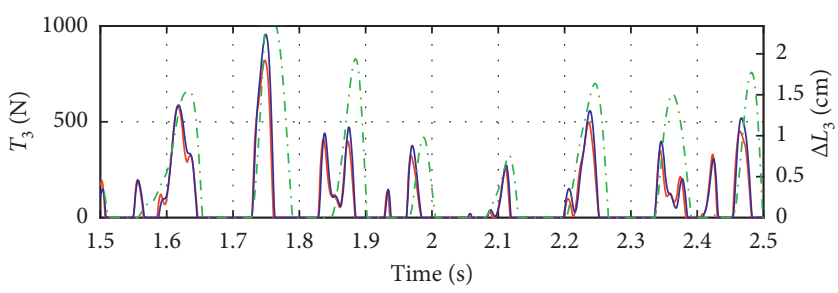

(c)

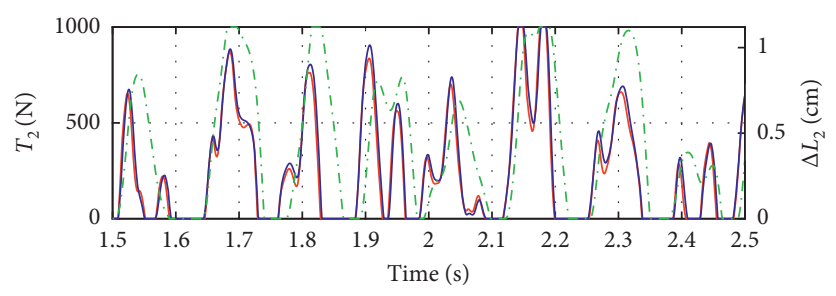

(b)

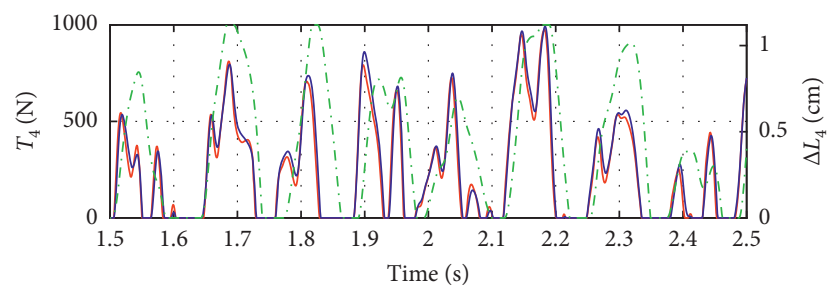

(d)

Figure 12: Tensions and longitudinal deformations of the cables. The red curve is the ideal $T_{i}$ without considering the inertial constraint in the CACS; the blue curve is the $T_{i}$ under $\tau=1 /(160 \pi)$; the green curve is the $T_{i}$ under $\tau=1 /(16 \pi)$.

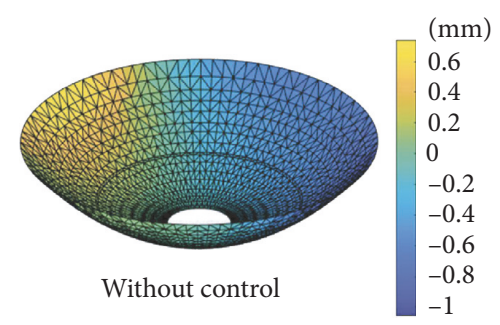

(a)

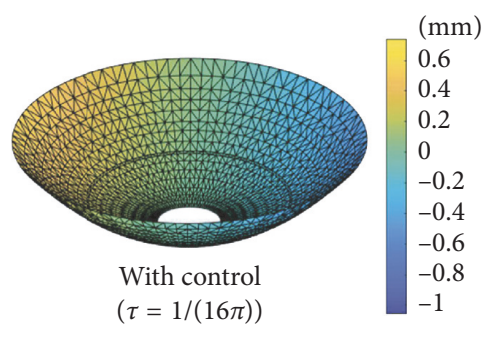

(b)

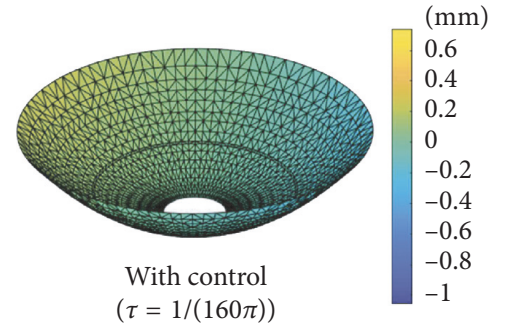

(c)

Figure 13: SVD in $W$ direction at $3 \mathrm{~s}$.

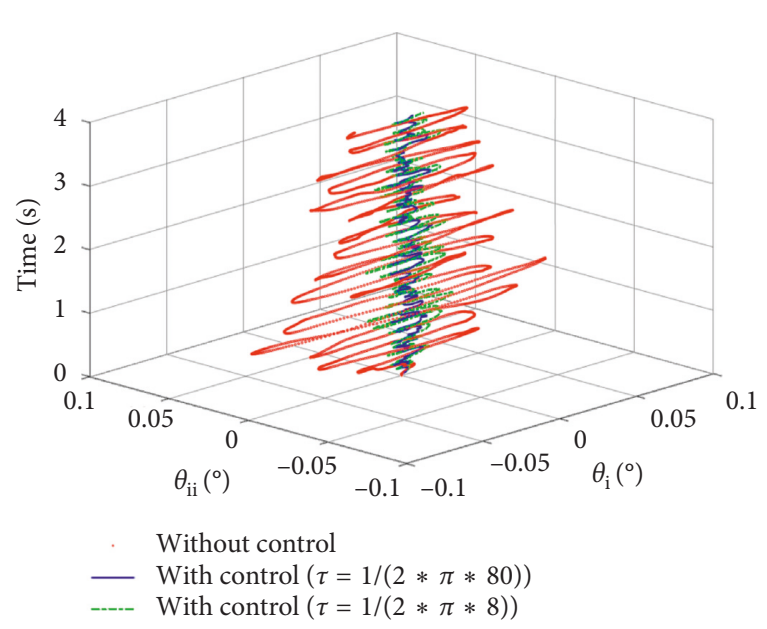

Figure 14: PEs without control and with control.

close to the ideal $T_{i}$; with the lower servo bandwidth of the CACS under $\tau=1 /(16 \pi)$, the phase of the $T_{i}$ has a certain lag with respect to the ideal $T_{i}$. The longitudinal deformation of the cable, as shown in Figure 12, is obtained by the following formula in which the cable is assumed as a linear spring [25]:

$$
\begin{aligned}
\Delta L_{i} & =\frac{T_{i}}{k_{c i}}, \\
k_{c i} & =\frac{E A_{r}}{L_{i}},
\end{aligned}
$$

where $L_{i}$ is the length of the cable.

Therefore, the $T_{i}$ under $\tau=1 /(160 \pi)$ has a better control effect on SVD and PE compared to the $T_{i}$ under $\tau=1 /(16 \pi)$, as shown in Figures 13 and 14. In the end, the RMS PEs with the adaptive system under the absence of inertial constraint, $\tau=1 /(160 \pi)$ and $\tau=1 /(16 \pi)$, are reduced by $80.26 \%$, $79.44 \%$, and $66.45 \%$ compared with the uncontrolled situation, respectively.

\section{Conclusion}

In this paper, a novel antenna monitoring and control system is proposed to suppress transient wind disturbance. From the initial study, the following conclusions are drawn:

(1) In the new adaptive system, the accelerometers placed on the reflector are used to obtain the vibration state of SVD caused by wind forces and 
tensions of the cables; the four-cableactuator mechanism with a circular slide track is designed to suppress unknown transient wind forces.

(2) To monitor the vibration, the first equivalent transformation is carried out to simplify the complex problem of identifying unknown spatial distribution forces including wind forces and cable tensions into identifying the equivalent forces of some nodes; based on the acceleration measurement and a low-order model, the identified equivalent forces and the vibration state are estimated successively in an unbiased minimum-variance way.

(3) To control the vibration, the second equivalent transformation is carried out to get cable tensions from the state forces for suppressing the vibration; the state forces are determined by the vibration state and the optimal gain of the LQR.

(4) Since the wind conditions are ever changing in the real environment, the loads acting on the antenna are different under different wind directions or speeds. To further improve control performance under random wind disturbance, how to optimize the system parameters and enhance the system robustness are key problems to be addressed in the future work.

(5) This method is currently applicable to the situation where rotating shaft of antenna are locked, such as the communication between the antenna and the geostationary spacecraft. In order to achieve an accurate tracking motion of the antenna, flexible multibody dynamics research of the system still needs to be done.

\section{Appendix}

\section{Unbiased Minimum-Variance Input and State Estimation Method}

Initial quantities:

$$
\begin{aligned}
& \text { initial state prediction: } \mathbf{x}_{0 \mid-1}, \\
& \text { initial state prediction covariance: } \mathbf{P}_{0 \mid-1}^{x} \text {. }
\end{aligned}
$$

Estimation of unknown inputs:

$$
\begin{aligned}
\widetilde{\mathbf{R}}_{k} & =\mathbf{C}_{a} \mathbf{P}_{k \mid k-1}^{x} \mathbf{C}_{a}^{T}+\mathbf{R}, \\
\mathbf{M}_{k} & =\left(\mathbf{D}_{e 1}^{T} \widetilde{\mathbf{R}}_{k}^{+} \mathbf{D}_{e 1}\right)^{+} \mathbf{D}_{e 1}^{T} \widetilde{\mathbf{R}}_{k}^{+}, \\
\widehat{\mathbf{F}}_{k}^{\prime} & =\mathbf{M}_{k}\left(\mathbf{a}_{k}-\mathbf{C}_{a} \mathbf{x}_{k \mid k-1}\right), \\
\mathbf{P}_{k}^{F} & =\left(\mathbf{D}_{e 1}^{T} \widetilde{\mathbf{R}}_{k}^{+} \mathbf{D}_{e 1}\right)^{+}
\end{aligned}
$$

Measurement update:

$$
\begin{aligned}
\mathbf{K}_{k} & =\mathbf{P}_{k \mid k-1}^{x} \mathbf{C}_{a}^{T} \widetilde{\mathbf{R}}_{k}^{+}, \\
\mathbf{P}_{k \mid k}^{x} & =\mathbf{P}_{k \mid k-1}^{x}-\mathbf{K}_{k}\left(\widetilde{\mathbf{R}}_{k}-\mathbf{D}_{e 1} \mathbf{P}_{k}^{F} \mathbf{D}_{e 1}^{T}\right) \mathbf{K}_{k}^{T}, \\
\mathbf{P}_{k}^{x F} & =\left(\mathbf{P}_{k}^{F x}\right)^{T}=-\mathbf{K}_{k} \mathbf{D}_{e 1} \mathbf{P}_{k}^{F}, \\
\mathbf{x}_{k \mid k} & =\mathbf{x}_{k \mid k-1}+\mathbf{K}_{k}\left(\mathbf{a}_{k}-\mathbf{C}_{a} \mathbf{x}_{k \mid k-1}-\mathbf{D}_{e 1} \widehat{\mathbf{F}}_{k}^{\prime}\right) .
\end{aligned}
$$

Time update:

$$
\begin{aligned}
\mathbf{x}_{k+1 \mid k} & =\mathbf{A} \mathbf{x}_{k \mid k}+\mathbf{B}_{e 1} \widehat{\mathbf{F}}_{k}^{\prime}, \\
\mathbf{P}_{k+1 \mid k}^{x} & =\left[\mathbf{A}, \mathbf{B}_{e 1}\right]\left[\begin{array}{cc}
\mathbf{P}_{k \mid k}^{x} & \mathbf{P}_{k}^{x F} \\
\mathbf{P}_{k}^{F x} & \mathbf{P}_{k}^{F}
\end{array}\right]\left[\begin{array}{c}
\mathbf{A}^{T} \\
\mathbf{B}_{e 1}^{T}
\end{array}\right]+\mathbf{Q} .
\end{aligned}
$$

\section{Data Availability}

The data used to support the findings of this study are available from the corresponding author upon request.

\section{Conflicts of Interest}

The authors declare that they have no conflicts of interest.

\section{Acknowledgments}

This work was supported by the National Natural Science Foundation of China under Grant nos. 51705387 and 51575419, the China Postdoctoral Science Foundation under Grant no. 2017M613078, the Nature Science Basic Research Plan in Shaanxi province of China under Grant no. 2019JQ-269, the Fundamental Research Funds for the Central Universities under Grant no. XJS190401, and the National 111 Project under Grant no. B14042.

\section{Supplementary Materials}

The supplementary material is a simulation animation of this paper. The transient wind field of the antenna is located in the upper left corner. The deformation of the antenna reflector under wind disturbance is in the lower left corner. The pointing error caused by the deflection of the reflector under wind disturbance is on the right side; the controlled situation is under the absence of inertial constraint in the CACS. (Supplementary Materials)

\section{References}

[1] J. Zhang, J. Huang, S. Wang, and C. Wang, "An active pointing compensator for large beam waveguide antenna under wind disturbance," IEEE/ASME Transactions on Mechatronics, vol. 21, no. 2, pp. 860-871, 2016.

[2] W. Gawronski and K. Souccar, "Control systems of the large millimeter telescope," IEEE Antennas and Propagation Magazine, vol. 47, no. 4, pp. 41-49, 2005. 
[3] Y. H. Hong, H.-K. Kim, and H. S. Lee, "Reconstruction of dynamic displacement and velocity from measured accelerations using the variational statement of an inverse problem," Journal of Sound and Vibration, vol. 329, no. 23, pp. 49805003, 2010.

[4] H. P. Gavin, R. Morales, and K. Reilly, "Drift-free integrators," Review of Scientific Instruments, vol. 69, no. 5, pp. 2171-2175, 1998.

[5] H. S. Lee, Y. H. Hong, and H. W. Park, "Design of an FIR filter for the displacement reconstruction using measured acceleration in low-frequency dominant structures," International Journal for Numerical Methods in Engineering, vol. 82, no. 4, pp. 403-434, 2010.

[6] N. Ukita, H. Ezawa, S. Onodera, and M. Saito, "Wind-induced pointing errors and surface deformation of a 10 - $\mathrm{m}$ submillimeter antenna," in Proceedings of SPIE, vol. 7733, p. 77331D, San Diego, CA, USA, June-July 2010.

[7] R. Snel, J. Mangum, and J. Baars, "Study of the dynamics of large reflector antennas with accelerometers," IEEE Antennas and Propagation Magazine, vol. 49, no. 4, pp. 84-101, 2007.

[8] W. Gawronski, "Antenna control systems: from PI to H/ sub /spl infin//," IEEE Antennas and Propagation Magazine, vol. 43, no. 1, pp. 52-60, 2001.

[9] D. Qiu, M. Sun, Z. Wang, Y. Wang, and Z. Chen, "Practical wind-disturbance rejection for large deep space observatory antenna," IEEE Transactions on Control Systems Technology, vol. 22, no. 5, pp. 1983-1990, 2014.

[10] J. Zhang, J. Huang, L. Qiu, and R. Song, “Analysis of reflector vibration-induced pointing errors for large antennas subject to wind disturbance: evaluating the pointing error caused by reflector deformation," IEEE Antennas and Propagation Magazine, vol. 57, no. 6, pp. 46-61, 2015.

[11] J. Zhang, J. Huang, P. B. Zhao, W. Liang, Y. Zhang, and C. Wang, "A pointing error analysis model for large reflector antennas under wind disturbance," Proceedings of the Institution of Mechanical Engineers, Part C: Journal of Mechanical Engineering Science, vol. 233, no. 6, pp. 1939-1951, 2019.

[12] J. Zhang, J. Huang, P. Zhao, W. Liang, X. Tang, and C. Wang, "Compensation of vibration-induced pointing error caused by wind disturbances in large antenna," Proceedings of the Institution of Mechanical Engineers, Part C: Journal of Mechanical Engineering Science, vol. 232, no. 23, pp. 4289-4300, 2018.

[13] J. Zhang, J. Huang, P. Zhao, W. Liang, and C. Wang, "Antenna control systems for flexible structure under a wind load," Proceedings of the Institution of Mechanical Engineers, Part C: Journal of Mechanical Engineering Science, vol. 233, no. 9, pp. 3050-3059, 2019.

[14] S. Gillijns and B. De Moor, "Unbiased minimum-variance input and state estimation for linear discrete-time systems with direct feedthrough," Automatica, vol. 43, no. 5, pp. 934-937, 2007.

[15] E. Lourens, C. Papadimitriou, S. Gillijns, E. Reynders, G. De Roeck, and G. Lombaert, "Joint input-response estimation for structural systems based on reduced-order models and vibration data from a limited number of sensors," $\mathrm{Me}$ chanical Systems and Signal Processing, vol. 29, pp. 310-327, 2012.

[16] K. Maes, A. W. Smyth, G. De Roeck, and G. Lombaert, "Joint input-state estimation in structural dynamics," Mechanical Systems and Signal Processing, vol. 70-71, pp. 445-466, 2016.

[17] K. Maes, E. Lourens, K. Van Nimmen, E. Reynders, G. De Roeck, and G. Lombaert, "Design of sensor networks for instantaneous inversion of modally reduced order models in structural dynamics," Mechanical Systems and Signal Processing, vol. 52-53, pp. 628-644, 2015.

[18] Q. B. Jin and J. Huang, "Active vibration control of large space flexible slewing truss using cable actuator with input saturation," International Journal of Robust and Nonlinear Control, vol. 28, no. 2, pp. 504-518, 2018.

[19] W. G. Perkins and R. S. Porter, "Solid-state extrusion of nylons 11 and 12: processing, morphology and properties," Journal of Materials Science, vol. 16, no. 6, pp. 1458-1470, 1981.

[20] W. Liang, J. Huang, J. Zhang, X. Tang, and Y. Zhang, "Reconstruction of vibration-deformation-induced pointing error via optimized acceleration measurement points for large reflector antennas," Proceedings of the Institution of $\mathrm{Me}$ chanical Engineers, Part C: Journal of Mechanical Engineering Science, vol. 233, no. 10, pp. 3417-3428, 2019.

[21] W. Gawronski, Modeling and Control of Antennas and Telescopes, Springer, New York, NY, USA, 2004.

[22] C. S. Wang, L. Xiao, W. Wang et al., "An adjustment method for active reflector of large high-frequency antennas considering gain and boresight," Research in Astronomy and Astrophysics, vol. 17, no. 5, p. 043, 2017.

[23] D. S. Li, H. N. Li, and C. P. Fritzen, "The connection between effective independence and modal kinetic Energy methods for sensor placement," Journal of Sound and Vibration, vol. 305, no. 4-5, pp. 945-955, 2007.

[24] E. Lourens and D. J. M. Fallais, "Full-field response monitoring in structural systems driven by a set of identified equivalent forces," Mechanical Systems and Signal Processing, vol. 114, pp. 106-119, 2019.

[25] S. Q. Fang, D. Franitza, M. Torlo, F. Bekes, and M. Hiller, "Motion control of a tendon-based parallel manipulator using optimal tension distribution," IEEE-ASME Transactions on Mechatronics, vol. 9, no. 3, pp. 561-568, 2004. 


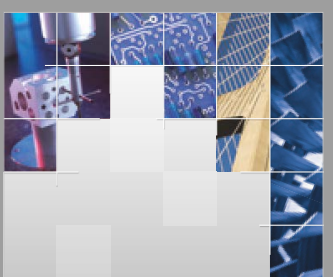

\section{Enfincering}
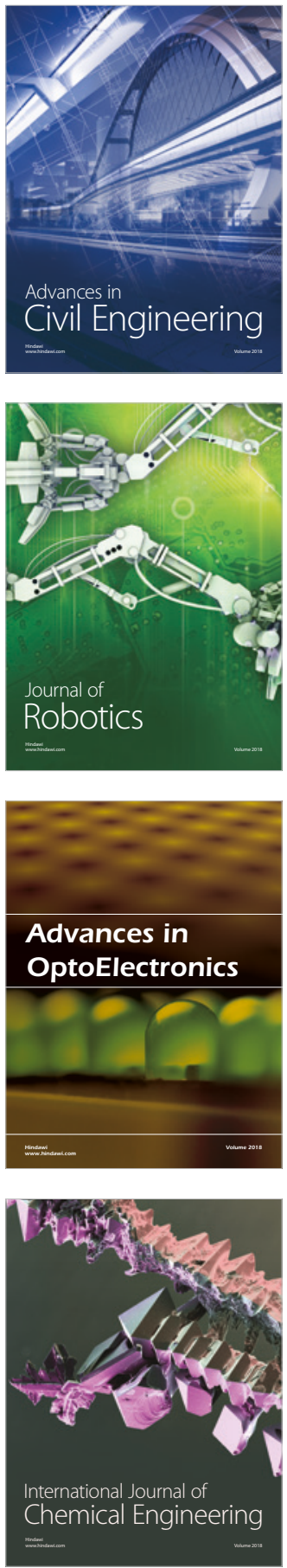

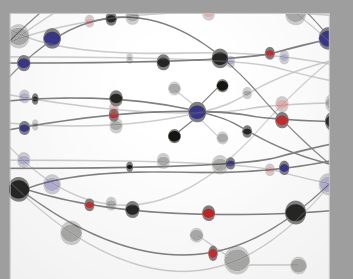

\section{Rotating \\ Machinery}

The Scientific World Journal

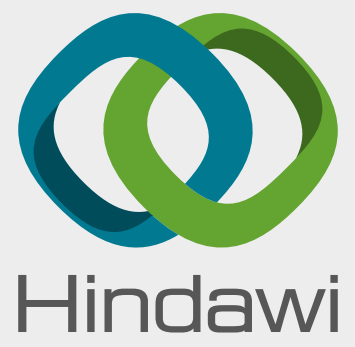

Submit your manuscripts at

www.hindawi.com
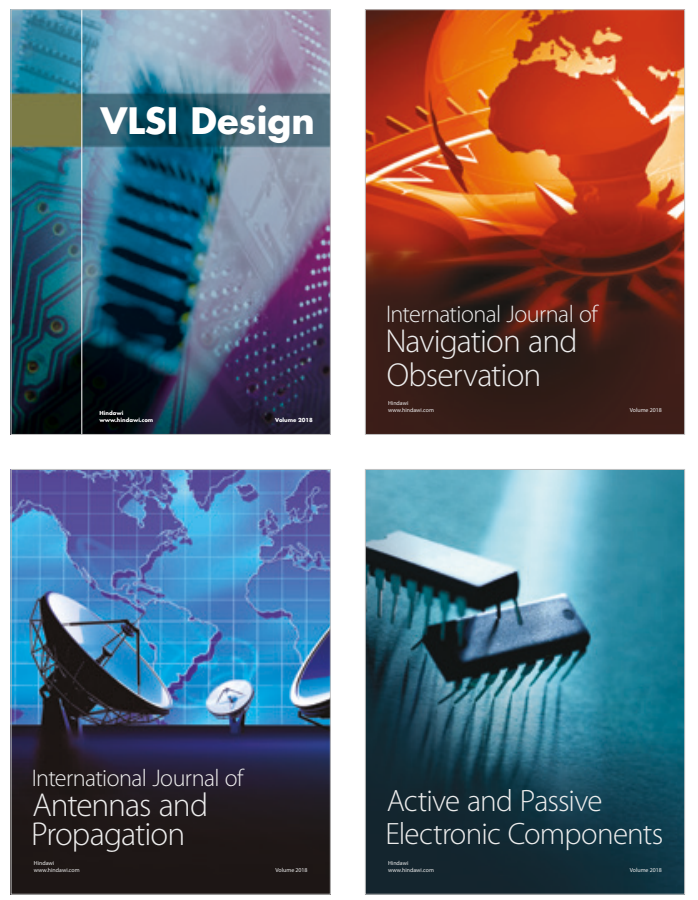
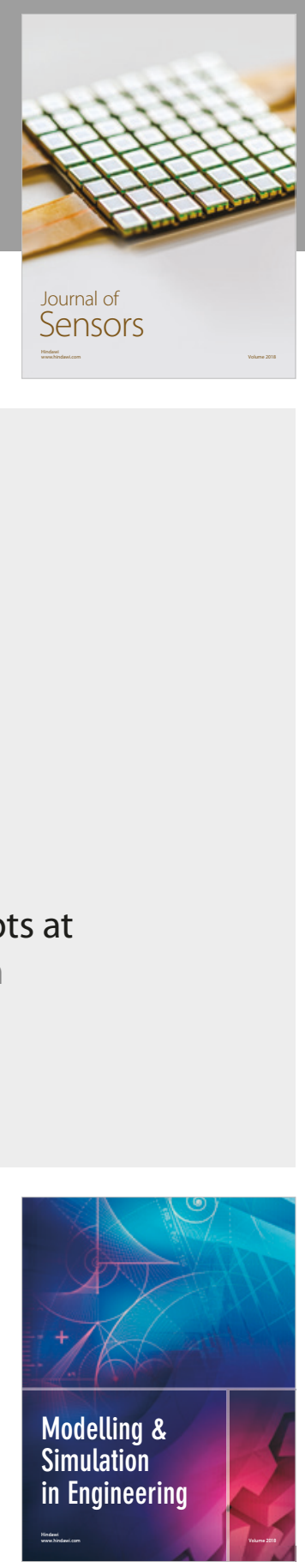

\section{Advances \\ Multimedia}
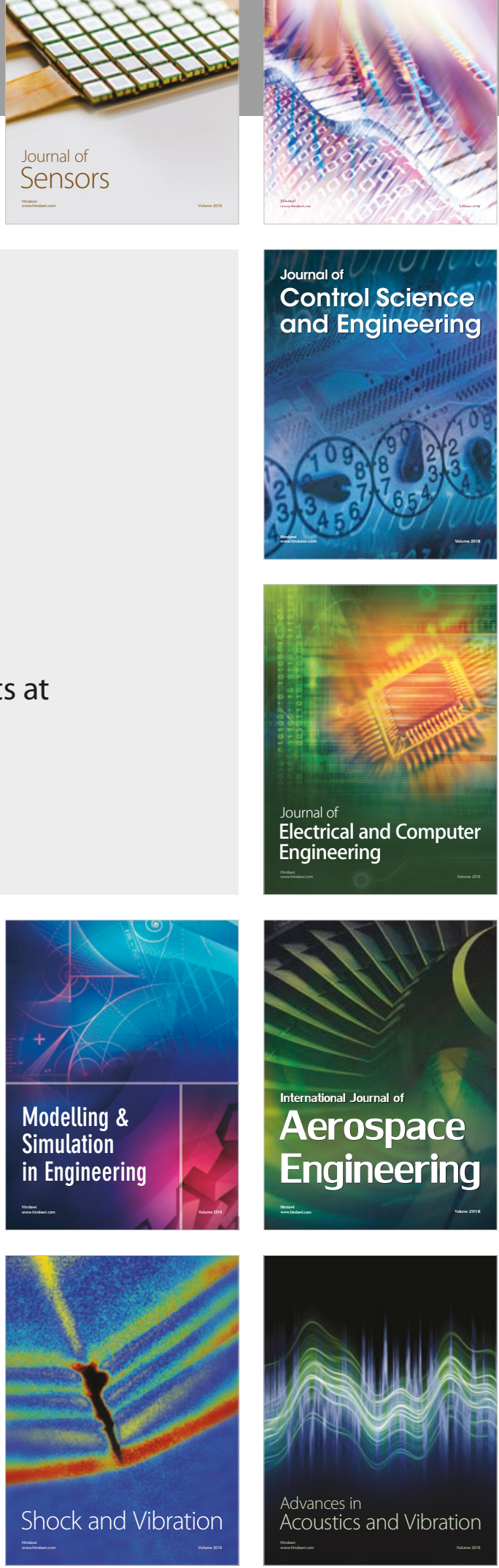